\title{
Stereotactic body radiotherapy combined with transarterial chemoembolization for hepatocellular carcinoma with portal vein tumor thrombosis
}

\author{
JINGBO KANG , QING NIE, RUI DU, LIPING ZHANG, JUN ZHANG, QILIANG LI, JIANGUO LI and WENJIE QI \\ Department of Radiotherapy, Navy General Hospital, Beijing 100048, P.R. China
}

Received March 30, 2013; Accepted July 26, 2013

DOI: $10.3892 /$ mco.2013.196

\begin{abstract}
The purpose of this study was to evaluate the clinical efficacy, toxicity and adverse effects of stereotactic body radiotherapy (SBRT) combined with transarterial chemoembolization (TACE) in patients with advanced hepatocellular carcinoma (HCC) with portal vein tumor thrombosis (PVTT). A total of 101 patients diagnosed with primary HCC with PVTT were enrolled in this study and were randomly divided into three groups as follows: group A, 34 patients treated with $\gamma$-SBRT followed by TACE; group B, 37 patients treated with TACE followed by $\gamma$-SBRT; and group C, 30 patients treated with $\gamma$-SBRT alone. The effective response rate for the entire patient sample was $87.1 \%(88 / 101)$ following a 3 -month treatment. The differences in the response rate, survival rate, $\alpha$-fetoprotein level restoration rate and rate of improvement of abdominal distention and discomfort between groups $\mathrm{A}$ and $\mathrm{B}$ were not statistically significant $(\mathrm{P}>0.05)$. However, the rates of groups $\mathrm{A}$ and $\mathrm{B}$ were higher compared to those of group $\mathrm{C}(\mathrm{P}<0.05)$. The exacerbation rate of liver function in group A was lower compared to that in group $\mathrm{B}(\mathrm{P}<0.05)$, although it exhibited no statistically significant difference from that in group $\mathrm{C}(\mathrm{P}>0.05)$. No severe radiation-related complications were reported during the follow-up period. The combination of $\gamma$-SBRT and TACE was shown to be a relatively effective local treatment for primary HCC patients with PVTT. Compared to $\gamma$-SBRT followed by TACE and $\gamma$-SBRT alone, TACE followed by $\gamma$-SBRT may exert a negative effect on liver function. These results suggested that the combination of TACE and $\gamma$-SBRT may be considered a relatively effective, safe and feasible treatment method for primary HCC patients with PVTT, although TACE followed by $\gamma$-SBRT may negatively affect liver function.
\end{abstract}

Correspondence to: Professor Jingbo Kang, Department of Radiotherapy, Navy General Hospital, 6 Fu Cheng Road, Beijing 100048, P.R.China

E-mail: kangjingbo_bj@sina.cn

Key words: primary hepatocellular carcinoma, portal vein tumor thrombosis, stereotactic body radiotherapy, $\gamma$-knife, transcatheter arterial chemoembolization

\section{Introduction}

Hepatocellular carcinoma (HCC) is ranked as the third most common cause of cancer-related mortality worldwide, with $>500,000$ cases diagnosed annually (1). Epidemiologic evidence demonstrated that HCC is frequently associated with hepatitis B virus (HBV) infection in China (2). Hepatic resection and transplantation, resulting in 5-year survival rates of $30-70 \%$, were shown to be beneficial for $<20 \%$ of patients (3). Therefore, local tumor control is crucial for patients with unresectable HCC prior to liver transplantation. Over the last decade, transarterial chemoembolization (TACE), radiofrequency ablation, Yttrium-90 microsphere embolization and percutaneous ethanol injection have been commonly used for improving local tumor control (4). However, these regimens exhibit limitations regarding lesion size, location, number and distribution.

The incidence of portal vein tumor thrombosis (PVTT) is $30-40 \%$ in HCC patients. PVTT is a poor prognostic factor, which may lead to wide dissemination of tumor cells throughout the liver, severely compromising liver function (5). TACE has become one of the major treatment options for patients with unresectable HCC, although its efficacy remains controversial (6-7). Alternative treatment options, such as intra-arterial chemotherapy and immunotherapy, have not demonstrated survival benefits (8-9). Therefore, an efficient way to improve local control for HCC with PVTT is required.

The application of traditional radiotherapy in the treatment of HCC is limited, due to radiation-induced liver disease (RILD) and the low tolerance of the liver to radiotherapy (10). Recent advances in radiotherapy techniques, including three-dimensional conformal radiotherapy (3D-CRT) and image-guided radiotherapy, have enabled the delivery of a higher radiation dose to the tumor rather than to the surrounding normal tissues (11). Stereotactic body radiotherapy (SBRT), a 3D-CRT irradiation technique developed to optimize target dose delivery and normal tissue sparing, is emerging as a viable treatment option for HCC patients. Accumulating evidence demonstrated that SBRT has become a treatment option for local tumor control of primary and secondary malignancies of the liver. Promising responses to high-dose radiotherapy to partial liver volume in patients with unresectable HCC have been observed (12-14). The combination of TACE with SBRT has been previously used in the management of HCC 
patients with PVTT (15-16). Despite its potential advantages, the combination of TACE with SBRT to treat advanced HCC with PVTT is poorly documented, with only a few available case reports (15). In this study, we retrospectively investigated patients with primary $\mathrm{HCC}$ and evaluated the response rate and toxicity of SBRT alone and SBRT combined with TACE for advanced HCC with PVTT.

\section{Materials and methods}

Ethics statement. The patients completed a questionnaire and provided written informed consent. The study was performed according to the Helsinki Declaration and the samples were processed following approval of the written consent statement by the Ethics Committee of the Navy General Hospital.

Patients. Between February, 2004 and March 2008, a consecutive case series of HCC patients with major vascular invasion (PVTT and inferior vena cava tumor thrombus), treated by TACE and $\gamma$-SBRT at the Department of Radiotherapy, Navy General Hospital, Beijing, were retrospectively investigated. The eligibility criteria were as follows: patient age of $\geq 18$ years, definite diagnosis of primary HCC with major vascular invasion (PVTT and inferior vena cava tumor thrombus), class A or B liver function according to the Child-Pugh classification and absence of previous radiotherapy to the liver. In all the patients, the diagnosis of HCC was based on histological confirmation or a characteristic tumor appearance on at least two imaging studies [including dynamic computed tomography (CT) scans, positron emission tomography-CT and dynamic contrast-enhanced magnetic resonance imaging (MRI) scans] and the presence of risk factors including HBV and HCV infection and cirrhosis. The presence and extent of PVTT were assessed by multiphase dynamic CT scans with a routine slice thicknesses of $5 \mathrm{~mm}$, using the following criteria: i) a low-attenuation intraluminal filling defect adjacent to the primary tumor during the portal phase and ii) an enhanced inner side of the filling defect during the arterial phase (17).

Study design and procedures. The study protocol was approved by the Ethics Committee of our hospital. A total of 120 patients were enrolled in the study and SPSS software, version 12.0 (SPSS Inc., Chicago, IL, USA) was used to randomly assign the observations into 3 equal sized groups (seed=85,123), with each group including 40 patients. Finally, 101 patients met the inclusion criteria. After picking out the ineligible observations, the group assignment was as follows: group A, 34 patients treated with $\gamma$-SBRT followed by TACE within the next 2-3 weeks; group B, 37 patients treated with TACE followed by $\gamma$-SBRT within the next 2-3 weeks; and group C, 30 patients treated with $\gamma$-SBRT alone.

Treatment process. The Seldinger method was used to puncture and cannulate the proper hepatic artery through the femoral artery. Following placement of a catheter, a mixture comprising 3-10 ml of iodized oil (Lipiodol; Laboratoires André Guerbet, Aulnay-sous-Bois, Paris, France), 600-1,000 mg fluorouracil and $30-50 \mathrm{mg}$ cisplatin was infused using a catheter placed directly into the right or left hepatic artery, with emboli- zation performed using gelatin sponge cubes (Gelfoam; Upjohn, Kalamazoo, MI, USA). The treatment was repeated at 6-8-week intervals until complete disappearance of the viable intrahepatic tumor, provided that hepatic function was preserved. TACE (median number of sessions, 2; range, 1-4) was performed prior to or following SBRT in the patients of group A or B, whereas patients in group $\mathrm{C}$ were treated with SBRT alone. The interval between TACE and SBRT was at least 4 weeks.

SBRT was planned following identification of PVTT at initial presentation or follow-up imaging and was initiated 2-3 weeks prior to or following TACE. Tumors imaged on the planning triphasic CT and/or MRI enhancing large vessel thromboses were included within the gross target volume [gross tumor volume (GTV)]. An 8-mm margin around the GTV within the liver and non-enhancing thromboses was included within the clinical target volume. The planning target volume (PTV) margins were individualized (minimum, $5 \mathrm{~mm}$ ), as previously described (18). The PTV around the GTV was the primary target $\left(\mathrm{PTV}_{\text {Primary }}\right)$, whereas the PTV around the clinical target volume $\left(\mathrm{PTV}_{\text {Secondary }}\right)$ was a secondary target. Conformal planning was used, with 3-10 coplanar or non-coplanar beams of 6-18 MV, with up to 3 segments within each field.

The dose-volume histogram for the liver minus the GTV (referred to as liver) was used to estimate the risk of RILD and to allocate the dose to the PTV $\mathrm{Primary}_{\text {. The dose to }}$

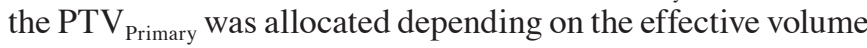
of irradiated liver $\left(\mathrm{V}_{\mathrm{eff}}\right)$ (Appendix, online only) and the uninvolved liver volume with a maximum dose of $60 \mathrm{~Gy}$. The target dose to PTV $\mathrm{P}_{\text {Secondary }}$, containing possible microscopic disease, was 24 Gy. SBRT was delivered in 6 fractions distributed over 2 weeks, usually on alternate days. The maximum permitted dose to $0.5 \mathrm{ml}$ of the esophagus, stomach, duodenum or bowel was $30 \mathrm{~Gy}$. The maximum dose to the spinal cord was $27 \mathrm{~Gy}$ and to the heart $40 \mathrm{~Gy}$. Efforts were made to minimize the irradiation of normal tissues.

Evaluation. Patients underwent abdominal CT scans 1 month following the completion of SBRT, after which time the tumor response was assessed at 2-3-month intervals. Tumor responses were classified according to the modified World Health Organization Response Evaluation Criteria (11) as follows: complete response (CR), complete disappearance of the irradiated tumor; partial response $(\mathrm{PR}),>50 \%$ reduction in tumor volume; stable disease (SD), decrease of $<50 \%$ or $>25 \%$ in tumor volume; and progressive disease (PD), $>25 \%$ increase in tumor volume.

The response of PVTT to $\gamma$-SBRT was evaluated by serial CT scans performed 3 months after the completion of radiotherapy. The product of the largest perpendicular diameter of the tumor thrombus was calculated and compared to the initial value and the PVTT response was defined as follows: CR, complete disappearance of the PVTT; PR, $\geq 50 \%$ decrease in the diameter of the thrombus; SD, $<50 \%$ decrease or $<25 \%$ increase in the diameter of the thrombus; and PD, $\geq 25 \%$ increase in the diameter of the thrombus. The objective response was estimated based on the combined number of patients with $\mathrm{CR}$ and $\mathrm{PR}$ and the progression-free rate of PVTT included patients with CR, PR and SD. The sum of CR and PR was defined as the objective response rate. 
Table I. Patient characteristics.

\begin{tabular}{|c|c|c|c|c|c|}
\hline Variables & Total & Group A (n) & Group B (n) & Group C (n) & P-value \\
\hline Gender & & & & & 0.892 \\
\hline Male & 69 & 23 & 25 & 21 & \\
\hline Female & 32 & 11 & 12 & 9 & \\
\hline Age & & & & & 0.232 \\
\hline Range & $19-79$ & $19-69$ & $25-70$ & $20-79$ & \\
\hline Median age & 53 & 50 & 53 & 55 & \\
\hline Child-Pugh grade & & & & & 0.743 \\
\hline Grade A & 67 & 23 & 25 & 19 & \\
\hline Grade B & 34 & 11 & 12 & 11 & \\
\hline$\alpha$-fetoprotein & & & & & 0.174 \\
\hline$>500 \mu \mathrm{g} / 1$ & 36 & 15 & 12 & 9 & \\
\hline $20-500 \mu \mathrm{g} / 1$ & 39 & 9 & 16 & 14 & \\
\hline$<20 \mu \mathrm{g} / \mathrm{l}$ & 26 & 10 & 9 & 7 & \\
\hline Tumor stage & & & & & 0.708 \\
\hline $\mathrm{IIb}$ & 67 & 22 & 23 & 22 & \\
\hline IIIa & 34 & 11 & 13 & 10 & \\
\hline Maximum lesion diameter & & & & & 0.981 \\
\hline$<5 \mathrm{~cm}$ & 32 & 12 & 11 & 9 & \\
\hline$\geq 5 \mathrm{~cm}<10 \mathrm{~cm}$ & 49 & 17 & 18 & 14 & \\
\hline$\geq 10 \mathrm{~cm}$ & 20 & 5 & 7 & 8 & \\
\hline No. of lesions & & & & & 0.793 \\
\hline 1 & 57 & 19 & 21 & 17 & \\
\hline 2 & 35 & 12 & 12 & 11 & \\
\hline$\geq 3$ & 9 & 3 & 4 & 2 & \\
\hline Location of embolism & & & & & 0.886 \\
\hline Trunk & 34 & 11 & 13 & 10 & \\
\hline Branches & 67 & 23 & 24 & 20 & \\
\hline Clinical manifestations & & & & & 1.000 \\
\hline Abdominal distension & 53 & 17 & 20 & 16 & \\
\hline Jaundice & 25 & 9 & 10 & 6 & \\
\hline Ascites & 25 & 8 & 9 & 8 & \\
\hline
\end{tabular}

Toxicity was graded using the National Cancer Institute Common Toxicity Criteria for Adverse Events, version 3.0. Any grade 4 or 5 hepatic or gastrointestinal toxicity or thrombocytopenia occurring within 1 month of SBRT, or RILD requiring treatment in the absence of disease progression within 3 months following SBRT was considered as dose-limiting toxicity (17). For Child-Pugh liver function determination, the international normalized ratio was considered to be stable in patients requiring warfarin treatment.

The European Organization for Research and Treatment of Cancer QLQ-HCC18 questionnaire was used to assess abdominal distension, jaundice and ascites as the life quality dimension of patients.

Statistical analysis. The overall and progression-free survival rates of PVTT were estimated from the date of detection of PVTT to the date of death or last follow-up and to the date of PVTT progression, respectively. The probability of cumulative survival was calculated according to the Kaplan-Meier method. The univariate and multivariate analyses were performed using a Cox proportional hazards models. Variables with $\mathrm{P}<0.05$ by univariate analysis were selected for multivariate analysis. $\mathrm{P}<0.05$ was considered to indicate a statistically significant difference. All the aforementioned analyses were performed using SPSS statistical software, version 12.0 (SPPS Inc., Chicago, IL, USA).

\section{Results}

Patient characteristics. The characteristics of the patients and the tumors prior to treatment are summarized in Table I. The majority of the subjects were male $(68.3 \%)$, with a median age of 53 years (range, 19-79 years). Invasion of the main portal vein was observed in all the patients and of the unilateral first-order branch of the portal vein in 34 patients $(33.7 \%)$. Radiotherapy was initiated in all the patients 2-3 weeks prior 
Table II. Tumor and portal vein tumor thrombus (PVTT) response rates.

\begin{tabular}{|c|c|c|c|c|c|c|}
\hline Cases & CR & PR & SD & PD & $\mathrm{RR}(\mathrm{CR}+\mathrm{PR})$ & P-value \\
\hline Tumor response (n) & & & & & & NS \\
\hline Group A (34) & 9 & 21 & 2 & 2 & $88.2 \%(30 / 34)$ & \\
\hline Group B (37) & 11 & 22 & 2 & 2 & $89.2 \%(33 / 37)$ & \\
\hline Group C (30) & 9 & 16 & 3 & 2 & $83.3 \%(25 / 30)$ & \\
\hline Total (101) & 29 & 59 & 7 & 6 & $87.1 \%(88 / 101)$ & \\
\hline PVTT response (n) & & & & & & NS \\
\hline Group A (34) & 7 & 18 & 4 & 5 & $73.5 \%(25 / 34)$ & \\
\hline Group B (37) & 6 & 20 & 6 & 5 & $70.3 \%(26 / 37)$ & \\
\hline Group C (30) & 5 & 15 & 5 & 5 & $66.7 \%(20 / 30)$ & \\
\hline Total (101) & 18 & 53 & 15 & 15 & $70.3 \%(71 / 101)$ & \\
\hline
\end{tabular}

CR, complete response; PR, partial response; $\mathrm{SD}$, stable disease; PD, progressive disease; RR, response rate; NS, non-significant.

Table III. Median survival time, local control rate and survival rate.

\begin{tabular}{|c|c|c|c|c|c|}
\hline & \multirow{2}{*}{$\begin{array}{l}\text { Median survival time } \\
\text { (months) }\end{array}$} & \multicolumn{2}{|c|}{ Local control rate } & \multicolumn{2}{|c|}{ Survival rate } \\
\hline & & 1-year & 2-year & 1-year & 2-year \\
\hline Group A & 15 & $55.9 \%(19 / 34)$ & $29.4 \%(10 / 34)$ & $58.8 \%$ & $29.4 \%$ \\
\hline Group B & 15 & $48.6 \%(18 / 37)$ & $24.3 \%(9 / 37)$ & $54.1 \%$ & $27.0 \%$ \\
\hline Group C & 12 & $43.3 \%(12 / 30)$ & $20.0 \%(6 / 30)$ & $50.0 \%$ & $23.3 \%$ \\
\hline
\end{tabular}

to or following TACE. The median radiotherapy dose was 40.2 Gy (range, 21-60 Gy). Since different doses per fraction were used, the biologically effective dose and equivalent dose in 2-Gy fractions, as the $\mathrm{a} / \mathrm{b}$ ratio of 10 , were also calculated. A small proportion of the patients $(16.7 \%)$ received radiotherapy with a target volume that included the whole HCC and PVTT.

Overall and PVTT response rates. Of the 101 patients assessed for tumor response at 6 months after diagnosis of PVTT, 29 patients (28.7\%) achieved a CR, $59(58.4 \%)$ achieved a PR and 7 patients $(8.3 \%)$ had SD, yielding an objective response rate of $87.1 \%$ and a progression-free rate of $95.4 \%$. As shown in Table II, the response rates of the patients in groups A $(88.2 \%, 30 / 34)$ and B $(89.2 \%$, $33 / 37)$ were higher compared to those in group C $(83.3 \%$, $25 / 30$ ), although the difference was not statistically significant. Of the 101 patients assessed for response of PVTT, 18 patients $(17.8 \%)$ achieved a CR, $53(52.5 \%)$ achieved a PR, 15 (14.9\%) had SD and 15 patients (14.8\%) had PD at 2-3 months after the completion of SBRT. The objective response rate and the progression-free rate of PVTT was 70.3 and $85.1 \%$, respectively (Table II). The response rates of the patients in groups A $(73.5 \%, 25 / 34)$ and B $(70.3 \%, 26 / 37)$ were higher compared to those in group C $(66.7 \%, 20 / 30)$, although the difference was not statistically significant. An example of follow-up CT images of patients who achieved a $\mathrm{CR}$ at 12 months after treatment with combined TACE and radiotherapy is presented in Fig. 1.
Survival analysis and predictors of survival. The median follow-up period was 15 months (range, 6-42 months). The median survival time was 17,15 and 12 months in groups A, $\mathrm{B}$ and $\mathrm{C}$, respectively. The 1- and 2-year local control rates were $55.9 \%(19 / 34)$ and $29.4 \%(10 / 34), 48.6 \%(18 / 37)$ and $24.3 \%(9 / 37)$ and $43.3 \%(12 / 30)$ and $20.0 \%(6 / 30)$, respectively, in groups A, B and C (Table III). The 1- and 2-year survival rates were 58.8 and $29.4 \%, 54.1$ and $27.0 \%$ and 50.0 and $23.3 \%$, respectively, in groups A, B and C. Based on the log-rank (Mantel-Cox) test, the median survival time and the survival rate of groups $\mathrm{A}$ and $\mathrm{B}$ were higher compared to those of group $\mathrm{C}$, although the difference was not statistically significant $(P=0.298)$. There was no significant difference between groups $\mathrm{A}$ and $\mathrm{B}$. The survival curves of the three groups are presented in Fig. 2.

Improvement of survival quality. Abdominal distension, jaundice and ascites are the major complications affecting the quality of life of HCC patients. In this study, we evaluated the quality of life of patients at 3 months after $\gamma$-SBRT treatment. As shown in Table IV, abdominal discomfort and distension were alleviated in $69.1 \%(38 / 55)$ of the patients. Among the patients treated with TACE prior to $\gamma$-SBRT or TACE following $\gamma$-SBRT, 72.2\% (13/18) and 71.4\% (15/21), respectively, were relieved from abdominal discomfort and distension, which was higher compared to those treated with $\gamma$-SBRT alone, who exhibited a $62.5 \%(10 / 16)$ relief rate. There was no significant difference in the resolution of jaundice among the different 

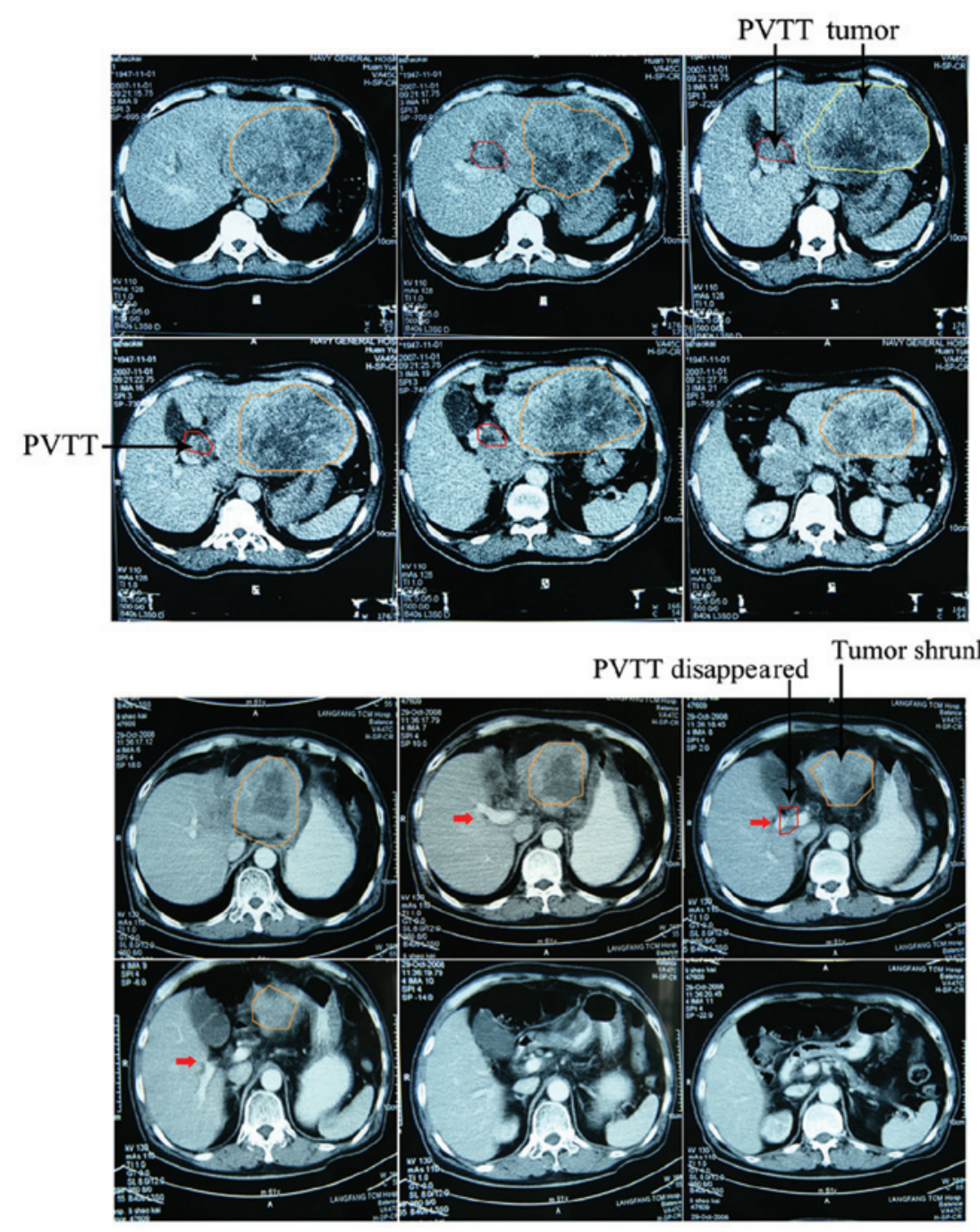

Figure 1. Example of follow-up computed tomography images.
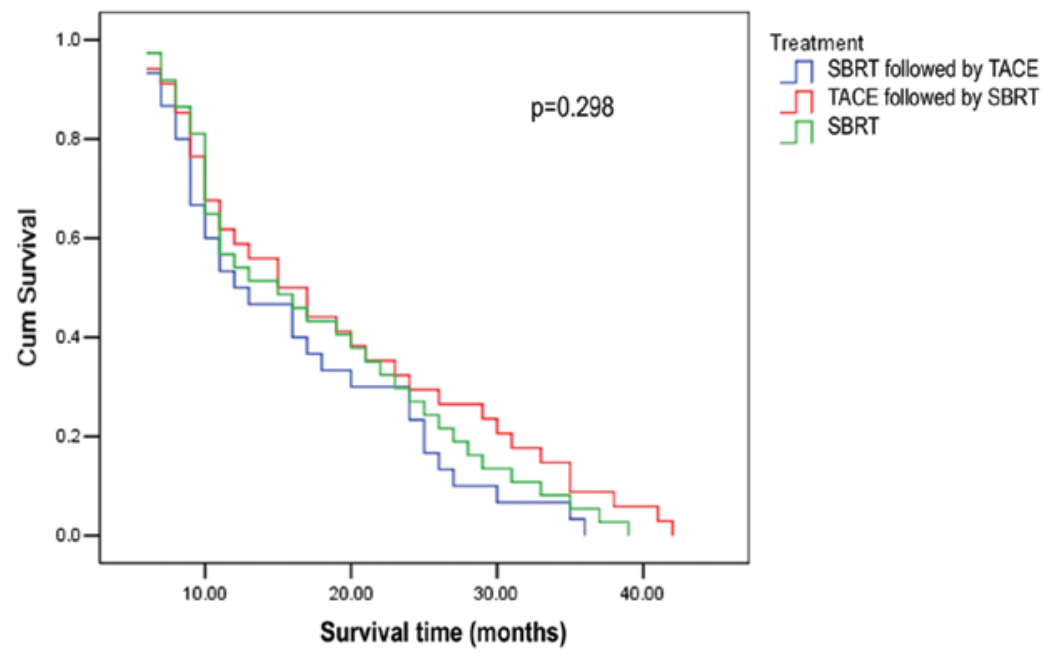

Figure 2. Survival curves of the three groups. TACE, transarterial chemoembolization; SBRT, stereotactic body radiotherapy; Cum, cumulative.

treatment groups. Among patients in group A, the jaundice and the icteric discoloration of the skin resolved in all 9 cases and the icterus index was decreased in 6 of the 9 patients. Of the
8 patients with a small or medium amount of ascitic fluid, the ascites resolved in 1 patient and was reduced in 4 patients. In group B, the jaundice and the icteric skin discoloration resolved 
Table IV. Improvement of life quality following radiotherapy.

\begin{tabular}{lccc}
\hline & $\begin{array}{c}\text { Relief of abdominal } \\
\text { discomfort and distension }\end{array}$ & Jaundice resolution & Ascites release \\
\hline Group A & $72.2 \%(13 / 18)$ & $66.6 \%(6 / 9)$ & $62.5 \%(5 / 8)$ \\
Group B & $71.4 \%(15 / 21)$ & $60.0 \%(6 / 10)$ & $55.6 \%(5 / 9)$ \\
Group C & $62.5 \%(10 / 16)$ & $50 \%(3 / 6)$ & $62.5 \%(5 / 8)$ \\
\hline
\end{tabular}

Table V. Adverse effects and exacerbation of liver function during 3 months after treatment.

Exacerbation grade of liver function

\begin{tabular}{|c|c|c|c|c|c|c|}
\hline $\begin{array}{l}\text { Adverse } \\
\text { effects }\end{array}$ & $\begin{array}{l}\text { Anorexia } \\
\text { and nausea }\end{array}$ & $\begin{array}{c}\text { Acute bone marrow } \\
\text { suppression (grade I+II) }\end{array}$ & $\begin{array}{l}\text { Grade A to } \\
\mathrm{B} \text { (case) }\end{array}$ & $\begin{array}{l}\text { Grade A to } \\
\mathrm{C} \text { (case) }\end{array}$ & $\begin{array}{c}\text { Grade B } \\
\text { to } \mathrm{C} \text { (case) }\end{array}$ & $\begin{array}{l}\text { Exacerbation } \\
\text { rate }\end{array}$ \\
\hline Group A & 13 & 14 & 8 & 1 & 2 & $32.4 \%(11 / 34)$ \\
\hline Group B & 15 & 17 & 10 & 3 & 2 & $40.5 \%(15 / 37)$ \\
\hline Group C & 11 & 9 & 7 & - & 2 & $30.0 \%(9 / 30)$ \\
\hline
\end{tabular}

in all 10 cases and the icterus index was decreased in 6 out of the 10 patients. The ascites resolved in 1 patient, was reduced in 4 cases and was aggravated in 3 cases, with a remission rate of $55.6 \%(5 / 9)$. In group $C$, the jaundice and the icteric skin discoloration resolved in all 6 cases and the icterus index was decreased in 3 patients. The ascites resolved in 1 patient and was reduced in 4 cases, with a remission rate of $62.5 \%(5 / 8)$.

Adverse effects. During the treatment, fatigue of various degrees, loss of appetite, nausea and other symptoms were observed in 13, 15 and 11 cases in groups A, B and C, respectively. There were no significant differences among the three groups. The incidence of grade I-II acute bone marrow suppression among the patients of group A (14/34, 41.2\%) and group B $(17 / 37,45.9 \%)$ was higher compared to that in group C (9/30, 29.6\%), although the difference was not statistically significant and they were all restored to normal following treatment. During the period between 1 and 3 months after treatment, exacerbation of liver function was observed in $32.4 \%(11 / 34), 40.5 \%(15 / 37)$ and $30.0 \%$ (9/30) of the patients in group $\mathrm{A}, \mathrm{B}$ and $\mathrm{C}$, respectively. The exacerbation rate of liver function in group B was higher compared to that in group A, with no statistically significant difference among the three groups. In detail, 8 cases deteriorated from grade A to B, 1 from $A$ to $C$ and 2 from $B$ to $C$ in group $A: 10$ cases deteriorated from grade $\mathrm{A}$ to $\mathrm{B}, 3$ from $\mathrm{A}$ to $\mathrm{C}$ and 2 from grade $\mathrm{B}$ to $\mathrm{C}$ in group $\mathrm{B}$; and 7 cases deteriorated from grade $\mathrm{A}$ to $\mathrm{B}$ and 2 from $B$ to $C$ in group $C$. Fever developed in 22 cases, with a body temperature of $\sim 38^{\circ} \mathrm{C}$ ). No other serious complications were reported during the follow-up period (Table V).

\section{Discussion}

The advantages of $\gamma$-SBRT in the treatment of HCC may be attributed to its ability to increase the radiation dose delivered to the tumor target while decreasing the irradiation of the surrounding normal tissues, which may result in improved local treatment and normal tissue protection (19-20). To the best of our knowledge, there is no available study on the sequence of $\gamma$-SBRT and TACE in patients with HCC with PVTT. Therefore, we compared the clinical efficacy, toxicity and adverse effects among the patients of three groups who received $\gamma$-SBRT followed by TACE, TACE followed by $\gamma$-SBRT, or $\gamma$-SBRT alone. The results demonstrated that the efficiency, control and survival rates in the two combined-modality groups were higher compared to those in the group who received $\gamma$-SBRT alone, whereas the liver function compromise in the patients who received TACE followed by $\gamma$-SBRT was more severe compared to that of the patients who were treated with $\gamma$-SBRT alone. These results demonstrated that TACE may result in liver function damage in HCC patients with PVTT, which should be considered during treatment planning. Therefore, if TACE is required first, due to the state of the disease, any changes in liver function should be monitored and the appropriate treatment planned accordingly.

TACE has been frequently used in patients with unresectable HCC. Izaki et al (21) applied TACE to treat patients with HCC combined with PVTT and the results demonstrated a median survival of 9.7 months, with accumulated 1-, 2- and 3 -year survival rates of 26.7, 13.3 and $13.3 \%$, respectively. Those results demonstrated that TACE is effective and safe for the treatment of PVTT. The effectiveness of TACE in PVTT may be attributed to the fact that PVTT receives its blood supply from the portal vein as well as the hepatic artery. A previous study by Sawrie et al (20) demonstrated that HCC was supplied by the hepatic artery in $24.4 \%$ patients, by the portal vein in $17.8 \%$ and by both in $57.8 \%$ patients. However, the long-term effects of the administration of TACE alone are not satisfactory, particularly in HCC patients with first-order branch or main portal vein invasion.

Radiotherapy has been shown to be effective to a certain extent on HCC with PVTT. Li et al (22) applied TACE followed by proton radiotherapy on 46 patients with HCC and PVTT and observed the effectiveness of the treatment. 
The experimental data suggested that TACE combined with proton radiotherapy was a novel, safe and effective treatment method. Seong et al (23) investigated the combination of CRT with TACE for HCC and reported a local control rate of $66 \%$. Ren et al (24) also reported that the thrombus remission rate following treatment by SBRT in patients with HCC and PVTT was $62.8 \%$. Therefore, if the patients with PVTT are first treated by $\gamma$-SBRT to reduce the size of PVTT and improve the blood supply of the portal vein, the addition of TACE may enhance the therapeutic effect and decrease the incidence of complications. Dang et al (25) used $\gamma$-SBRT to treat portal vein thrombosis and reported a CR of $21.1 \%$ and a PR of $26.3 \%$ in the $\gamma$-SBRT group, proving the effectiveness of $\gamma$-SBRT in the treatment of PVTT. Our results demonstrated that the changes in liver function in the group treated with $\gamma$-SBRT followed by TACE were similar to those in the group treated with $\gamma$-SBRT alone. This may be explained by the hypothesis that liver tumor cells exhibit an early response, whereas normal liver tissue and vessels exhibit a late response. This difference in the response to radiation may result in the early response occurring during irradiation or within the first few days or weeks after the treatment, whereas late response may occur after several months or years. Therefore, the first 2 weeks following $\gamma$-SBRT may be the time period of liver tumor cell response to the radiation, while the changes in vessels have not yet occurred and TACE performed during this period may not affect the therapeutic efficacy of the drugs.

Approximately $25-30 \%$ of the blood supply of normal liver tissue comes from the hepatic artery, which plays a role in cancer embolization for tumor control and shrinking in patients with partial obstruction of the portal vein, with drugs cycling in the hepatic artery. The theoretical evidence of the treatment option of TACE followed by $\gamma$-SBRT in patients with PVTT may be based on this physiological phenomenon. Performing $\gamma$-SBRT after TACE may be advantageous, as the reduction of the target volume by TACE may allow increase of radiation dose delivery to the target and/or decrease of the radiation damage of normal liver tissue. The administration of $\gamma$-SBRT after TACE may contribute to killing or inhibiting any residual tumor cells after TACE. The fact that prior administration of TACE affects liver function is reflected by the fact that the compromise of liver function in patients with PVTT who were treated with TACE followed by $\gamma$-SBRT was significantly different from that in patients treated by $\gamma$-SBRT alone.

Unlike linear accelertor-based SBRT that uses multiple fixed beams, $\gamma$-SBRT employs highly focused revolving radiation beams. These focused beams lead to the delivery of highly conformal doses to the targets, while greatly sparing normal tissues. As demonstrated in this study, $\gamma$-SBRT combined with TACE sequentially is an effective treatment option for HCC with PVTT. In particular, the remission effects of PVTT are comparable between the two combined-modality groups and the $\gamma$-SBRT-alone group, indicating that the remission may be the result of $\gamma$-SBRT.

In conclusion, the $\gamma$-knife-based SBRT combined with TACE is a relatively effective local treatment for patients with primary HCC and PVTT. Although there were no major radiation- related complications associated with these treatments, there were a few adverse reactions that were manageable with symptomatic treatment. The bone marrow suppression was not significantly different between the $\gamma$-SBRT alone and the $\gamma$-SBRT combined with TACE groups. Compared to $\gamma$-SBRT alone and $\gamma$-SBRT followed by TACE, TACE followed by $\gamma$-SBRT exerted a more prominent negative effect on liver function. If TACE followed by $\gamma$-SBRT is used, the relevant indicators of liver function should be closely monitored and, if required, appropriate treatment should be administered immediately.

\section{References}

1. Farazi PA and DePinho RA: Hepatocellular carcinoma pathogenesis: from genes to environment. Nat Rev Cancer 6: 674-687, 2006.

2. Yang HI, Lu SN, Liaw YF, et al: Hepatitis B e antigen and the risk of hepatocellular carcinoma. N Engl J Med 347: 168-174, 2002.

3. Parkin DM, Bray F, Ferlay J and Pisani P: Global cancer statistics, 2002. CA Cancer J Clin 55: 74-108, 2005.

4. Llovet JM: Updated treatment approach to hepatocellular carcinoma. J Gastroenterol 40: 225-235, 2005.

5. Cabibbo G, Enea M, Attanasio M, Bruix J, Craxi A and Camma C: A meta-analysis of survival rates of untreated patients in randomized clinical trials of hepatocellular carcinoma. Hepatology 51: 1274-1283, 2010.

6. Kim JH, Yoon HK, Kim SY, et al: Transcatheter arterial chemoembolization vs. chemoinfusion for unresectable hepatocellular carcinoma in patients with major portal vein thrombosis. Aliment Pharmacol Ther 29: 1291-1298, 2009.

7. Kothary N, Weintraub JL, Susman J and Rundback JH: Transarterial chemoembolization for primary hepatocellular carcinoma in patients at high risk. J Vasc Interv Radiol 18: 1517-1527, 2007.

8. Chung YH, Song IH, Song BC, et al: Combined therapy consisting of intraarterial cisplatin infusion and systemic interferon-alpha for hepatocellular carcinoma patients with major portal vein thrombosis or distant metastasis. Cancer 88: 1986-1991, 2000.

9. Obi S, Yoshida H, Toune R, et al: Combination therapy of intraarterial 5-fluorouracil and systemic interferon-alpha for advanced hepatocellular carcinoma with portal venous invasion. Cancer 106: 1990-1997, 2006.

10. Emami B, Lyman J, Brown A, et al: Tolerance of normal tissue to therapeutic irradiation. Int J Radiat Oncol Biol Phys 21: 109-122, 1991.

11. Krishnan S, Dawson LA, Seong J, et al: Radiotherapy for hepatocellular carcinoma: an overview. Ann Surg Oncol 15: 1015-1024, 2008.

12. Price TR, Perkins SM, Sandrasegaran K, et al: Evaluation of response after stereotactic body radiotherapy for hepatocellular carcinoma. Cancer 118: 3191-3198, 2012.

13. Facciuto ME, Singh MK, Rochon C, et al: Stereotactic body radiation therapy in hepatocellular carcinoma and cirrhosis: evaluation of radiological and pathological response. J Surg Oncol 105: 692-698, 2012.

14. Tse RV, Hawkins M, Lockwood G, et al: Phase I study of individualized stereotactic body radiotherapy for hepatocellular carcinoma and intrahepatic cholangiocarcinoma. J Clin Oncol 26: 657-664, 2008.

15. Choi BO, Choi IB, Jang HS, et al: Stereotactic body radiation therapy with or without transarterial chemoembolization for patients with primary hepatocellular carcinoma: preliminary analysis. BMC Cancer 8: 351, 2008.

16. Zhao M, Wang JP, Li W, et al: Comparison of safety and efficacy for transcatheter arterial chemoembolization alone and plus radiofrequency ablation in the treatment of single branch portal vein tumor thrombus of hepatocellular carcinoma and their prognosis factors. Zhonghua Yi Xue Za Zhi 91: 1167-1172, 2011 (In Chinese).

17. Dawson LA, Eccles $\mathrm{C}$ and Craig T: Individualized image guided iso-NTCP based liver cancer SBRT. Acta Oncol 45: 856-864, 2006.

18. Lawrence TS, Robertson JM, Anscher MS, Jirtle RL, Ensminger WD and Fajardo LF: Hepatic toxicity resulting from cancer treatment. Int J Radiat Oncol Biol Phys 31: 1237-1248, 1995.

19. Lo SS, Dawson LA, Kim EY, et al: Stereotactic body radiation therapy for hepatocellular carcinoma. Discov Med 9: 404-410, 2010. 
20. Sawrie SM, Fiveash JB and Caudell JJ: Stereotactic body radiation therapy for liver metastases and primary hepatocellular carcinoma: normal tissue tolerances and toxicity. Cancer Control 17: 111-119, 2010

21. Izaki K, Sugimoto K, Sugimura K and Hirota S: Transcatheter arterial embolization for advanced tumor thrombus with marked arterioportal or arteriovenous shunt complicating hepatocellular carcinoma. Radiat Med 22: 155-162, 2004.

22. Li Q, Zeng CJ, Wang Y:Interventional chemoembolization combined with proton radiotherapy for the treatment of hepatocellular carcinoma accompanied with portal cancerous thrombus. J Intervent Radiol 18: 278-280, 2009.
23. Seong J, Park HC, Han KH, et al: Clinical results of 3-dimensional conformal radiotherapy combined with transarterial chemoembolization for hepatocellular carcinoma in the cirrhotic patients. Hepatol Res 27: 30-35, 2003.

24. Ren B, Song JB, Wang XD, et al: Stereotactic radiotherapy for hepatocellular carcinoma with portal tumor thrombi. Chin J Hepatol 14: 308-309, 2006 (In Chinese).

25. Dang YZ, Zhang XC, Lu WL, et al: Body gamma knife and high intensity focused ultrasound (HIFU) for the treatment of portal vein tumor thrombosis of hepatocellular carcinoma. J Mod Oncol 18: 114-117, 2010. 